\title{
Factores determinantes de competitividad e integración organizacional: revisión sistemática exploratoria
}

\author{
Diana Romero-Suárez ${ }^{1 *}$, Vanessa Pertuz ${ }^{2}$ y Erick Orozco-Acosta ${ }^{3}$ \\ (1) Universidad Simón Bolívar, Barranquilla, Colombia. (correo-e: dianaromerosuarez@gmail.com) \\ (2) Universidad de Santander, Facultad de ingenierías, Grupo de Investigación Nuevas Tecnologías-UDES. Valledupar, \\ Colombia. (correo-e: van.pertuz@mail.udes.edu.co) \\ (3) Facultad de Ingenierías, Universidad Simón Bolívar, Barranquilla, Colombia. \\ (correo-e: eorozco15@unisimonbolivar.edu.co)
}

* Autor a quien debe ser dirigida la correspondencia.

Recibido Dic. 18, 2019; Aceptado Feb. 14, 2020; Versión final Mar. 17, 2020, Publicado Oct. 2020

\section{Resumen}

Este trabajo presenta una revisión sistemática exploratoria de la literatura, relacionada con los factores de competitividad e integración organizacional, desde el año 2013 hasta el año 2019. Esto se hace con información reportada por las principales bases bibliográficas. Los resultados muestran registros relacionados con las temáticas en Latinoamérica, Europa y Asia. Se evidencia un aumento en el número de artículos incluidos en las bases de datos frente a los temas de estudio. Se identificaron 23 factores de competitividad entre los que se destacan la estructura y cooperación entre empresas, la gestión del marketing y la implementación de tecnología innovadora en la producción. Igualmente, se establecen 17 factores que favorecen la integración organizacional, tales como la gobernanza de las relaciones estratégicas entre empresas, la coordinación intra-empresarial y la implementación de sistemas de gestión de calidad. Esta revisión contribuye con la generación de capacidades organizacionales para el desarrollo de la competitividad e integración de procesos.

Palabras clave: factores de competitividad; integración organizacional; revisión sistemática exploratoria

\section{Determining factors of competitiveness and organizational integration: scoping review}

\begin{abstract}
This work presents a systematic review of the literature related to the competitiveness and organizational integration from 2013 to 2019. This is done with information reported by the main bibliographic databases. The results show records related to competitiveness and organizational integration in Latin America, Europe, and Asia. There is evidence of an increase in the number of articles published during the period considered in the study. There are 23 competitiveness factors identified including the structure and cooperation between companies, marketing management, and the implementation of innovative technology in production. In addition, there are 17 factors that favor organizational integration such as the governance of strategic relationships between companies, intra-business coordination, and the implementation of quality management systems. This scoping review article contributes to the generation of organizational capacities for competitiveness development and process integration.
\end{abstract}




\section{INTRODUCCIÓN}

La competitividad y la integración organizacional son conceptos que han ganado importancia en diferentes sectores de la economía a nivel mundial, en efecto, en la literatura se identifican una importante cantidad de antecedentes relacionados con estas temáticas. A nivel mundial, se reconoce la importancia que generan las Micro, Pequeñas y Medianas Empresas (MIPYMES) para la economía, específicamente en términos de empleo, distribución del ingreso y producción. Considerando lo anterior, en la actualidad un importante número de investigaciones se concentran en la generación e implementación de estrategias o programas para la competitividad de las empresas, con el fin de fortalecer su dinámica dentro de los mercados nacionales e internacionales (Melgarejo et al., 2013).

Históricamente, la competitividad se reconoce como un atributo clave de las economías nacionales y sus sectores productivos, en los principales países del mundo (Labarca, 2007), lo cual ha promovido el interés por entender esta variable desde el punto de vista de las naciones y las empresas. A nivel de las naciones, la competitividad corresponde a la medida en que estas son capaces de producir bienes y servicios, que puedan ser comerciables en los mercados internacionales; con el fin de mantener la renta real de los ciudadanos de la nación, es decir, la capacidad de un país de posicionar sus productos en los mercados internacionales (Verner 2011). Por su parte, a nivel de empresas, la competitividad se define como la capacidad que tiene una organización de producir bienes o servicios y comercializarlos en condiciones óptimas de precio, calidad y oportunidad, a diferencia de sus rivales (Porter, 1987). De esta manera, la competitividad puede identificarse en la dimensión comercial y no comercial de una empresa. En la dimensión comercial se indica por medio de la rentabilidad, el porcentaje de exportación y participación en el mercado regional. Mientras que, en la dimensión no comercial, se asocia con la capacidad de competir a nivel global por una participación de mercado con costos eficientes y calidad de los bienes y servicios (Labarca, 2007).

De esta manera, la literatura presenta diversos factores que inciden en la competitividad de las empresas dentro de los que se incluyen, el conocimiento, como un activo fundamental en la actualidad para la generación del valor en las organizaciones (Ibarra et al., 2017), las relaciones laborales, los niveles de capacitación de la mano de obra y el acceso a recursos financieros (Saavedra y Tapia 2011). Estos factores inciden en las organizaciones para alcanzar el objetivo básico financiero de generar valor y conocimiento, traducido en la gestación de nuevos modelos de negocio (Saavedra y Tapia 2011). Así, la innovación es determinante en las empresas debido que fomenta el aprendizaje para el fortalecimiento de su posición competitiva en el mercado (Ahumada y Perusquia, 2016).

En concordancia con lo anterior, la competitividad puede ser analizada bajo la identificación de cuatro niveles: meta, macro, meso y micro (Esser et al., 1996; Hernández, 2001). El nivel meta aborda aspectos relacionados con la estructura política y económica orientada al desarrollo y los planes nacionales (Labarca, 2007). En el nivel macro se incluyen aspectos relevantes para la cadena productiva y económica del país (Rojas, 1999), el nivel micro analiza los factores internos de la empresa, tales como la productividad, los costos y la innovación, mientras que el nivel meso es la capacidad de fomentar y desarrollar los esfuerzos al nivel de la empresa (Messner, 1996).

En este contexto, la competitividad de las empresas se encuentra determinada por factores internos y externos (Leyva et al., 2018). En el marco del análisis de estos factores, es necesario destacar que la competitividad es un fenómeno complejo, en el que interactúan múltiples elementos como las prácticas de producción, organización y gestión empresarial, que generan ventajas competitivas. En consecuencia, la competitividad se convierte en un concepto dinámico, que modifica progresivamente los diferentes recursos internos y externos de las empresas (Navarro et al., 2018). Desde la perspectiva interna, las prácticas que afectan negativamente la competitividad de las empresas y que tienen origen ético son: la competencia desleal, el incumplimiento de la normatividad de las empresas, las condiciones económicas, el nivel de formación y los sobornos; asimismo, la pérdida de tiempo de los empleados y la falta de claridad con los clientes en el momento de realizar el proceso del servicio al cliente (Ramírez, 2016).

En referencia a la integración organizacional, es relevante destacar que las organizaciones son sociales y cooperativas, por lo que estas podrían integrarse con otras empresas con características diferenciales para mejorar su desempeño de un entorno empresarial (Montoya et al., 2008). Por lo tanto, la integración implica el trabajo conjunto de varias empresas que son antagónicas entre ellas, o la coordinación de los procesos de varios departamentos de una empresa (Todeva y Knoke, 2005). Como resultados de la integración, es posible el mejoramiento de las organizaciones en aspectos como: el establecimiento de ventajas de integración vertical $u$ horizontal, el desarrollo de estrategias coordinadas entre agentes, el incremento en el poder de negociación y la creación de nuevas combinaciones de negocios (Montoya et al., 2008). De esta manera, la integración se orienta al desarrollo de redes empresariales, mediante el impulso a la especialización y el aumento de la productividad (Hernández, 2001). 
Igualmente, es importante generar acciones cooperativas desde las empresas de diferentes sectores con el apoyo de instituciones académicas con el objeto de realizar proyectos orientados al desarrollo tecnológico y la innovación empresarial, considerando que a través de este tipo de iniciativas se logra un aprendizaje organizacional que incrementan las capacidades de innovación y beneficia a las empresas participantes mediante el fortalecimiento del capital relacional y el desarrollo regional (Becerra et al, 2013). Por medio del aprendizaje, las organizaciones tienen la posibilidad de crear asociaciones para afrontar de manera eficiente los retos del entorno en que se desempeñan. estas asociaciones pueden ser, externas mediante las alianzas; o internas, asumiendo que cada área o departamento de la empresa puede ser objeto de un proceso de integración organizacional (Todeva y Knoke, 2005). De esta manera, la integración de las firmas favorece las medidas de la productividad, la innovación, la formación de negocios y la cooperación (Mäkinen, 2001).

En este sentido, la integración organizacional, por medio del aprendizaje, puede generar un cambio de estructura e intercambio de información dentro de las empresas, que se genera mediante dos formas: intraorganizacional, a través de la interacción de los procesos, áreas, departamentos dentro de una misma empresa o inter-organizacional, correspondiente a la interacción con las demás empresas del entorno (Martínez, 2013). Frente al aspecto intra-organizacional, se hace referencia a las cadenas de suministros como una forma de integración, las cuales son definidas como sociedades formadas por áreas autónomas, que se unen para la solución de problemas (Chandra et al., 2001).

De acuerdo con lo planteado por Canales y Caldart (2017) existen cuatro factores que fomentan el desarrollo de la colaboración autónoma entre empresas: el sentido de urgencia a nivel de empresa, la existencia de la dirección estratégica directiva, los mecanismos de integración entre empresas y la cultura organizacional que promueve la integración. Mientras que Song et al., (2019) hace referencia a la capacidad de generar información por medio de la integración y crear integraciones en las organizaciones en el área de gestión logística afecta de manera significativa las cadenas de suministros.

De este modo, la competitividad y la integración organizacional impactan todas las áreas de la empresa con el fin de generar rentabilidad en los negocios (Khakpour et al., 2018). Así, los factores de competitividad e integración organizacional son procesos que se han fortalecido desde una perspectiva estratégica, mediante la estructuración de diferentes marcos conceptuales y de medición (Manrique y Vargas, 2017). La competitividad e integración organizacional incluyen la globalidad de la gestión organizacional, mediante la presencia de factores entre los que se encuentran: la tecnología (Vargas et al., 2016), gobierno (Cao y You, 2017), Calidad (Cao y You, 2017), relaciones industriales (Tambade et al., 2019), enfoque estratégico (Pereira y Crispim, 2015), relaciones de mercado (Oaigen et al., 2013), innovación (Ryzhkova y Prosvirkin, 2015), marketing (Nakipova, 2013); entre otros, que hacen parte fundamental de los procesos de cada una de las empresas y sectores. Considerando lo anterior, el articulo presenta la revisión sistemática exploratoria de la literatura reciente, relacionada con los factores de competitividad e integración organizacional. Para tal efecto, se consideran las variables bibliométricas asociadas a cada artículo de investigación, con el objetivo de establecer los elementos que favorecen competitividad e integración en el contexto de las organizaciones.

\section{METODOLOGÍA}

En el presente artículo se considera la metodología de revisión sistemática exploratoria propuesta por Manchado et al., (2009), "la cual se caracteriza por una serie de peculiaridades que permiten la exploración de no solo lo que se ha hecho con un tema, sino que también muestra quien, donde y de qué manera se ha abordado el tema. De acuerdo a los resultados obtenidos en la respectiva revisión se pueden crear hipótesis sobre diversas preguntas de investigación, como también proponer ámbitos de estudio" p.15.

La revisión sistemática exploratoria presentada en el artículo tiene como objeto de identificar en la literatura científica los factores de competitividad e integración organizacional. Para el desarrollo de la metodología propuesta se consideraron las bases de datos EBSCO, Scopus, Emerald Insight y Web of Science (WOS). EI periodo de la revisión corresponde a el año 2013 hasta el mes de mayo del año 2019. Se utilizaron dos ecuaciones de búsqueda; "competitiveness factors" para la identificación de los artículos relacionados con la competitividad y "business integration" para recuperar los documentos referentes a la integración organizacional.

En la presente revisión sistemática explotaría se definieron los siguientes criterios de inclusión: a) periodo de estudio desde el año 2013 hasta el año 2019, b) documentos publicados en los siguientes idiomas: español, inglés o portugués, c) como tipo de documento se consideran solo artículos originales de investigación, d) artículos que en el resumen hagan referencia a los factores de competitividad o integración organizacional.

En referencia a la extracción de información, los registros recuperados que cumplieron con los criterios de inclusión en cada una de las bases de datos, se exportaron al programa Mendeley, posteriormente, se 
eliminaron los documentos duplicados. Luego, se sistematizó una base de datos en el programa Microsoft Excel con las siguientes variables: año, autores, titulo, país, revista de publicación, institución de filiación, palabras claves, objetivo, diseño metodológico, técnica de análisis de datos, instrumentos de recolección, resultados, pregunta de investigación y factores identificados.

Posteriormente, se realizó la lectura crítica de todos los documentos, analizando los resultados a partir de los variables identificadas, con el fin de identificar los factores de competitividad e integración organizacional. De cada artículo se obtienen distintos factores; por lo que se realiza la estimación de las frecuencias de cada uno de estos en la literatura analizada. De este modo, en la revisión se identifican los factores de competitividad e integración organizacional más referenciados en la literatura.

Finalmente, se presentan las redes bibliométricas para complementar el análisis de los factores de competitividad e integración organizacional identificados en la revisión sistemática exploratoria. Estas redes fueron construidas mediante el programa VOSviewer, a partir de los resultados de la búsqueda bibliográfica. Las redes bibliométricas permiten identificar los nodos asociados a los factores de competitividad e integración organizacional y corroborar que los factores identificados en la revisión se asocian con los términos claves de los artículos indexados en las bases de datos de corriente principal.

De acuerdo con la revisión de los conceptos frente a las variables de estudio, para efectos del presente artículo se analiza la competitividad desde el enfoque de las empresas, la cual se define como la capacidad que tiene una organización de optimizar sus procesos, generando una producción de bienes y servicios eficientes, centrada en la calidad del producto, con el fin de comercializar el producto final en mercados nacionales e internacionales, que le permita lograr una diferenciación con las empresas competidoras.

Asimismo, a nivel organizacional, la integración es un proceso que se puede generar de dos formas (Martinez, 2013): la primera, intra-organizacional, en donde se fomenta la asociación de todas las áreas y departamentos dentro de una empresa, generando el fortalecimiento de los procesos. La segunda, inter-organizacional, en la cual se genera la asociación de varias empresas de un mismo sector, con el fin de generar ventajas entre ellas y fomentar el desarrollo de sus procesos.

\section{RESULTADOS}

A partir de la aplicación los criterios de inclusión definidos en la metodología de la presente revisión sistemática exploratoria, se realizó la búsqueda orientada a identificar los artículos originales publicados desde el año 2013 hasta mayo del 2019 en las temáticas de competitividad e integración organizacional. A continuación, se resume el proceso de selección de los documentos para cada una de las variables de estudio, a partir de las diferentes bases de datos. Puntualmente, se detalla el número de registros obtenidos en la búsqueda inicial, así como los documentos resultantes de cada uno de los criterios establecidos. En total, la presente revisión incluye 68 documentos, 49 de los cuales corresponden a la variable competitividad y 19 registros a integración organizacional. La Figura 1 presenta el resultado de la revisión de documentos relacionados con la variable competitividad.
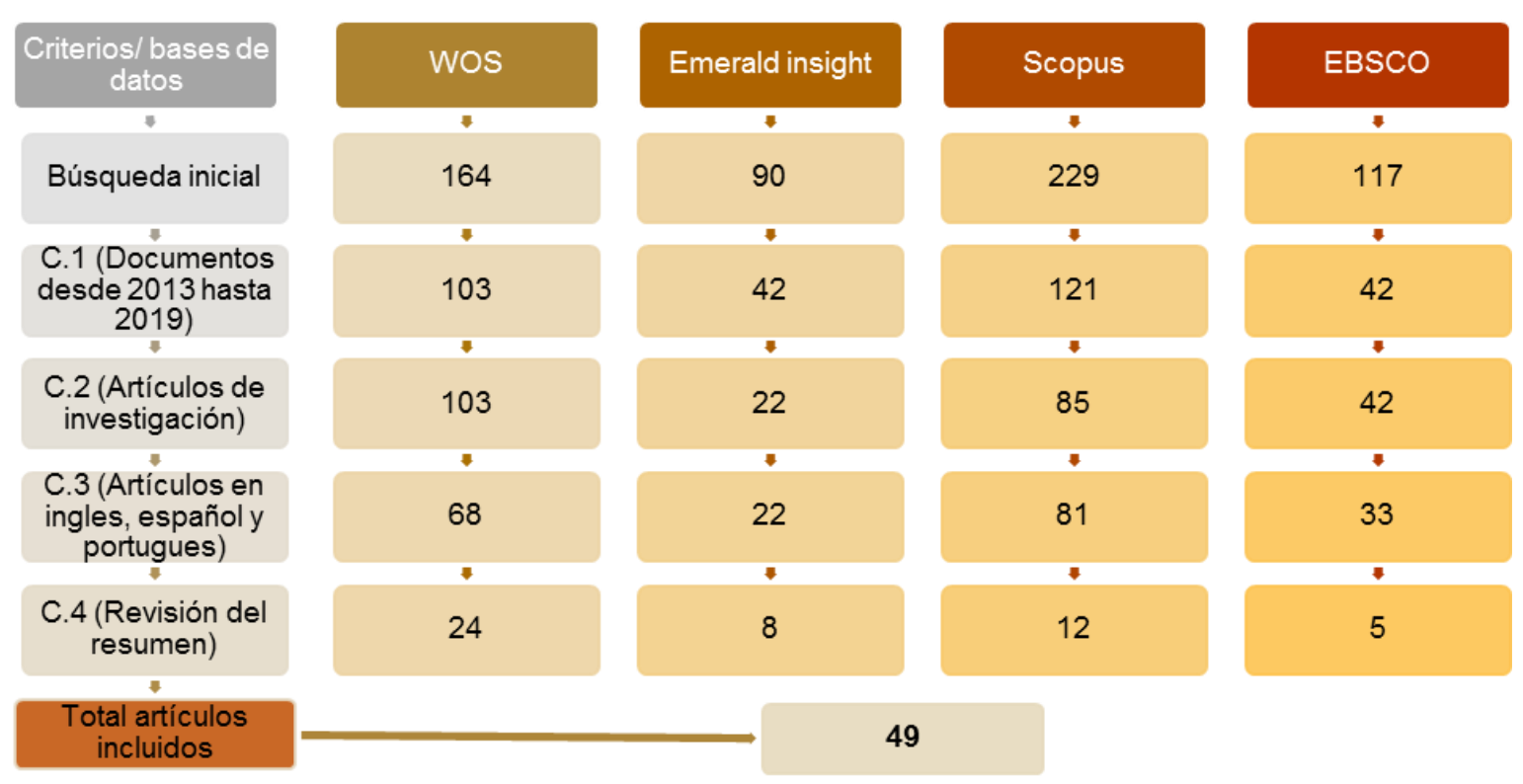

Fig. 1: Proceso de selección de la literatura relacionada con la competitividad 
De acuerdo con la Figura 1, en la búsqueda inicial correspondiente a la ecuación de competitividad se obtienen Ios siguientes registros: 164 documentos en la base de datos WOS, 90 documentos en Emerald Insight, 229 documentos se registran en Scopus y 117 en EBSCO. Del total de documentos identificados 308 corresponden a literatura publicada entre los años 2013 y 2019, y 252 registros se clasifican como artículos de investigación en las diferentes bases de datos. Conforme al tercer criterio de inclusión, se obtienen un total de 204 registros publicados en inglés, portugués y español. Finalmente, según el cuarto criterio de inclusión, se analizan los resúmenes de los documentos, para identificar si estos hacen referencia a los factores de competitividad. A partir de la revisión, un total de 49 artículos de investigación se incluyen la revisión sistemática exploratoria. Por su parte, la Figura 2 presenta el resultado de la revisión de documentos relacionados con la variable integración organizacional.

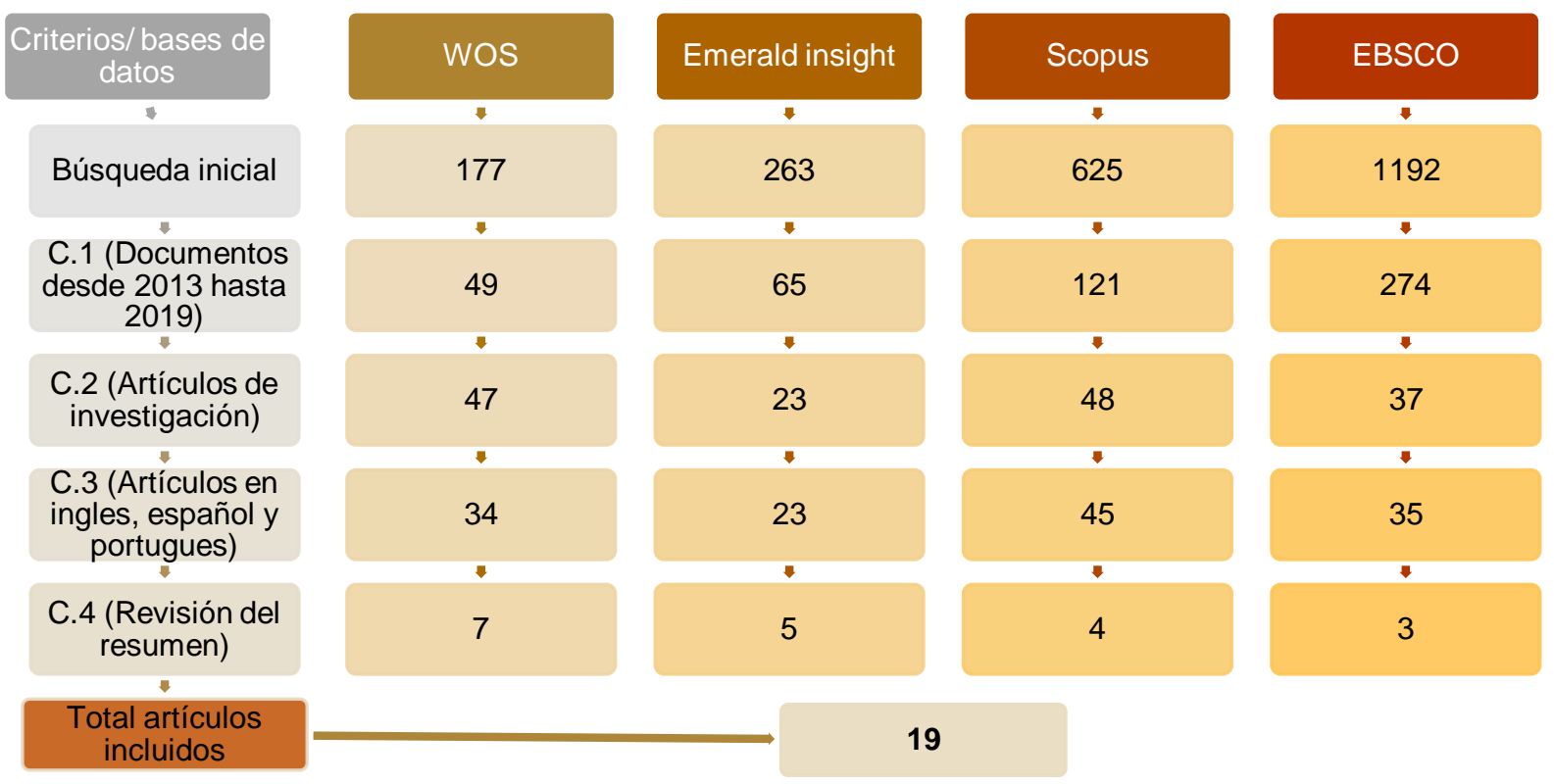

Fig. 2: Proceso de selección de la literatura relacionada con la integración organizacional

En concordancia con lo expuesto en la Figura 2, en la búsqueda inicial correspondiente a la ecuación de integración organizacional se obtienen los siguientes registros: 177 documentos en la base de datos WOS, 263 documentos en Emerald Insight, 625 documentos se registran en Scopus y 1192 en EBSCO. Del total de documentos identificados 509 corresponden a literatura publicada entre los años 2013 y 2019 , y 155 registros se clasifican como artículos de investigación en las diferentes bases de datos. De acuerdo con el tercer criterio de inclusión, se obtienen un total de 137 registros publicados en inglés, portugués y español. Finalmente, según el cuarto criterio de inclusión, se analizan los resúmenes de los documentos, para identificar si estos hacen referencia a los factores de integración organizacional. A partir de la revisión, un total de 19 artículos de investigación se incluyen la revisión sistemática exploratoria.

\section{Características de los estudios}

Se reporta literatura científica desde 2013 con tendencia creciente en el tiempo. Se evidencia la mayor producción científica en los años 2017 (21.2\%) y 2018 (17.7\%). Los artículos correspondientes al resto de los años incluidos en la presente revisión se distribuyen de la siguiente forma: 2013 (11.6\%), 2014, 2016 (14.35\%), 2015 (15.5\%) y 2019 (5,1\%). Es relevante indicar que para el año 2019 solo se consideran artículos publicados en el primer trimestre del año. Los países líderes en la producción de documentos científicos relacionados con la temática son: Brasil (19\%), China (9\%) y Colombia (8.5\%); seguidamente de India, Reino Unido y Rusia (7.5\%), en menor proporción España (5\%), Irán, Portugal, República Checa, USA y Ucrania (4\%), mientras que los demás países muestran un comportamiento de $2 \%$ o menos en la información estadística. De acuerdo con los resultados de la revisión frente al tema se establece que los factores de competitividad e integración organizacional corresponden a un importante campo de producción científica en Latinoamérica, Europa y Asia.

Adicionalmente, en el análisis de las variables bibliométricas se identifica un amplio número de revistas que publican artículos relacionados con las variables objeto de la presente revisión sistemática. Específicamente en competitividad, el $8 \%$, de los artículos incluidos en la revisión corresponden a la revista Competitiviness Review y el $6 \%$ en la revista Custos e Agronegocio. El $86 \%$ restante de las publicaciones analizadas 
corresponden a diversas fuentes como: Journal of Competitiviness, Journal of Information \& Knowledge Management, y Industrial Engineering \& Management Systems. Por su parte, para la variable de integración organizacional, un $10 \%$ de los artículos incluidos en la revisión provienen de la revista Benchmarking: an International Journal, los documentos restantes se incluyen en fuentes como: European Management Journal, Información Tecnológica y International Journal of Information Management.

\section{Identificación de los factores de competitividad e integración organizacional}

De acuerdo con los registros identificados durante el periodo de referencia de la revisión de literatura, a continuación, se presentan los factores de competitividad e integración organizacional, los cuales categorizan de acuerdo con el número de referencias en la literatura científica. Específicamente, la Tabla 1 presenta la frecuencia de los factores de competitividad.

Tabla 1: Frecuencia factores de competitividad identificados en la revisión

\begin{tabular}{|l|c|}
\hline \multicolumn{1}{|c|}{ Factor de competitividad } & Frecuencia \\
\hline Estructura y cooperación entre empresas & 35 \\
\hline Gestión del marketing & 28 \\
\hline Tecnología de producción innovadora & 27 \\
\hline Estrategia de gestión integrada y sistémica & 23 \\
\hline Políticas laborales y capital humano & 21 \\
\hline Gestión financiera & 21 \\
\hline Disponibilidad y costos de materias primas mediante la relación con proveedores & 21 \\
\hline Acceso a la tecnología & 19 \\
\hline Infraestructura física & 18 \\
\hline Relaciones con el gobierno y política industrial del estado & 16 \\
\hline Inversiones en investigación y Desarrollo (I+ D) y relación con la academia & 16 \\
\hline Capacitación y educación de los colaboradores & 14 \\
\hline Condiciones de la demanda & 12 \\
\hline Viabilidad y rentabilidad del modelo de negocio & 12 \\
\hline Condiciones económicas del entorno & 11 \\
\hline Responsabilidad social y medioambiental & 9 \\
\hline Ubicación en el territorio & 8 \\
\hline Asignación de procesos y recursos para innovación & 8 \\
\hline Logística de bienes y servicios & 8 \\
\hline Calidad de los bienes y servicios & 8 \\
\hline Exportaciones & 5 \\
\hline Capacidades directivas & 4 \\
\hline Comportamiento ético & 3 \\
\hline
\end{tabular}

Como resultado de la revisión sistémica exploratoria, se identifican los factores de competitividad con mayor número de referencias en los estudios. Al respecto, los factores más frecuentes en la literatura son: estructura y cooperación entre empresas, gestión del marketing y tecnología de producción innovadora. Mientras que los factores con menos referencias en la literatura consultada son: exportaciones, capacidades directivas y comportamiento ético. De otro lado, la Tabla 2 presenta la frecuencia de los factores de integración organizacional identificados en la revisión sistemática exploratoria.

De acuerdo con los resultados de la revisión presentados en la Tabla 2, los factores de integración organizacional con mayor número de referencias en la literatura corresponden a la gestión y gobernanza de las relaciones estratégicas entre empresas, la coordinación intra-empresarial, la implementación de sistemas de gestión de calidad y la gestión del talento humano. 
Tabla 2: Frecuencia factores de integración organizacional identificados en la revisión

\begin{tabular}{|l|c|}
\hline \multicolumn{1}{|c|}{ Factor de integración organizacional } & Frecuencia \\
\hline Gestión y gobernanza de las relaciones estratégicas entre empresas & 13 \\
\hline Gestión y coordinación intra-empresarial & 12 \\
\hline Implementación de sistemas de gestión de calidad & 10 \\
\hline Gestión del talento humano & 8 \\
\hline Eficacia y reingeniería de los procesos & 8 \\
\hline Gestión del conocimiento & 6 \\
\hline Estabilidad financiera y gestión de recursos & 5 \\
\hline Confianza entre las empresas & 3 \\
\hline Implementación de las tecnologías de la información y la comunicación & 3 \\
\hline Liderazgo de la alta gerencia & 3 \\
\hline Conocimiento del mercado & 3 \\
\hline Capacidad de negociación de la empresa & 3 \\
\hline Fortaleza de los vínculos entre empresas y capital social & 3 \\
\hline Estrategias de gestión de cartera & 2 \\
\hline Gestión de la cadena de valor & 2 \\
\hline Gestión de las partes interesadas & 2 \\
\hline Implementación de sistemas de planificación organizacional & 2 \\
\hline
\end{tabular}

\section{Redes bibliométrica de los factores de competitividad e integración organizacional}

En referencia a la red bibliométrica de factores de competitividad, construida a partir de la base de datos de Scopus, se identifican 6 clúster a partir de la concurrencia de palabras claves. El clúster 1 (rojo) corresponde al factor del comercio, en donde genera concurrencias los nodos de comercio internacional, manufactura y economía. El clúster 2 (verde) se encuentra el nodo de la innovación, el cual genera concurrencias con los nodos de competencia industrial, el sector servicio y el conocimiento. Adicionalmente, el clúster 3 (azul) corresponde al nodo de planeación estratégica, el cual genera concurrencia con los nodos de eficiencia, productividad y desarrollo sostenible. Por otro lado, El clúster 4 (amarillo) hace referencia al nodo de gestión de proyectos y ventaja competitiva. El clúster 5 (morado) corresponde al nodo de industria, con los nodos de comercio electrónico, economía industrial, marketing y gestión del conocimiento. Finalmente, para el clúster 6 (azul celeste) se encuentra el nodo del factor humano (Figura 3). En estos clústeres, se generó la identificación de los términos claves de las revistas indexadas, las cuales confirman los factores identificados en la revisión sistemática exploratoria.

Frente a la construcción de la red bibliométrica correspondiente a los factores de integración organizacional, se obtienen 6 clúster teniendo en cuenta la concurrencia de palabras claves de los artículos indexados en la base de datos Scopus: el clúster 1 (rojo), el cual corresponde al nodo de manufactura, en donde se genera concurrencia con los nodos de los costos, el comercio y el diseño de producto. El nodo de la integración, hace parte del clúster 2 (morado) creando concurrencia con los nodos de análisis de la información e interoperabilidad. En el clúster 3 (azul) se encuentra el nodo de servicios de información en donde se hayan concurrencias con los de servicios web e internet. De este modo, el nodo de comercio electrónico del clúster 4 (verde), se forman concurrencias con los nodos de sistemas de integración y la gestión de negocios. Así mismo, el nodo de la integración organizacional, la planificación de recursos empresariales y las cadenas de suministros hacen parte del clúster 4 y se presentan de manera independiente, por ser uno de los nodos con mayor relevancia en la red. En cuanto a los clústeres 5 (naranja) y 6 (azul celeste), presentan nodos con mínima concurrencia, y por lo tanto hace parte de esos factores que tienen menor relevancia y frecuencia en la literatura (Figura 4). Estos factores coinciden con los identificados en la revisión sistemática exploratoria de la literatura científica. 


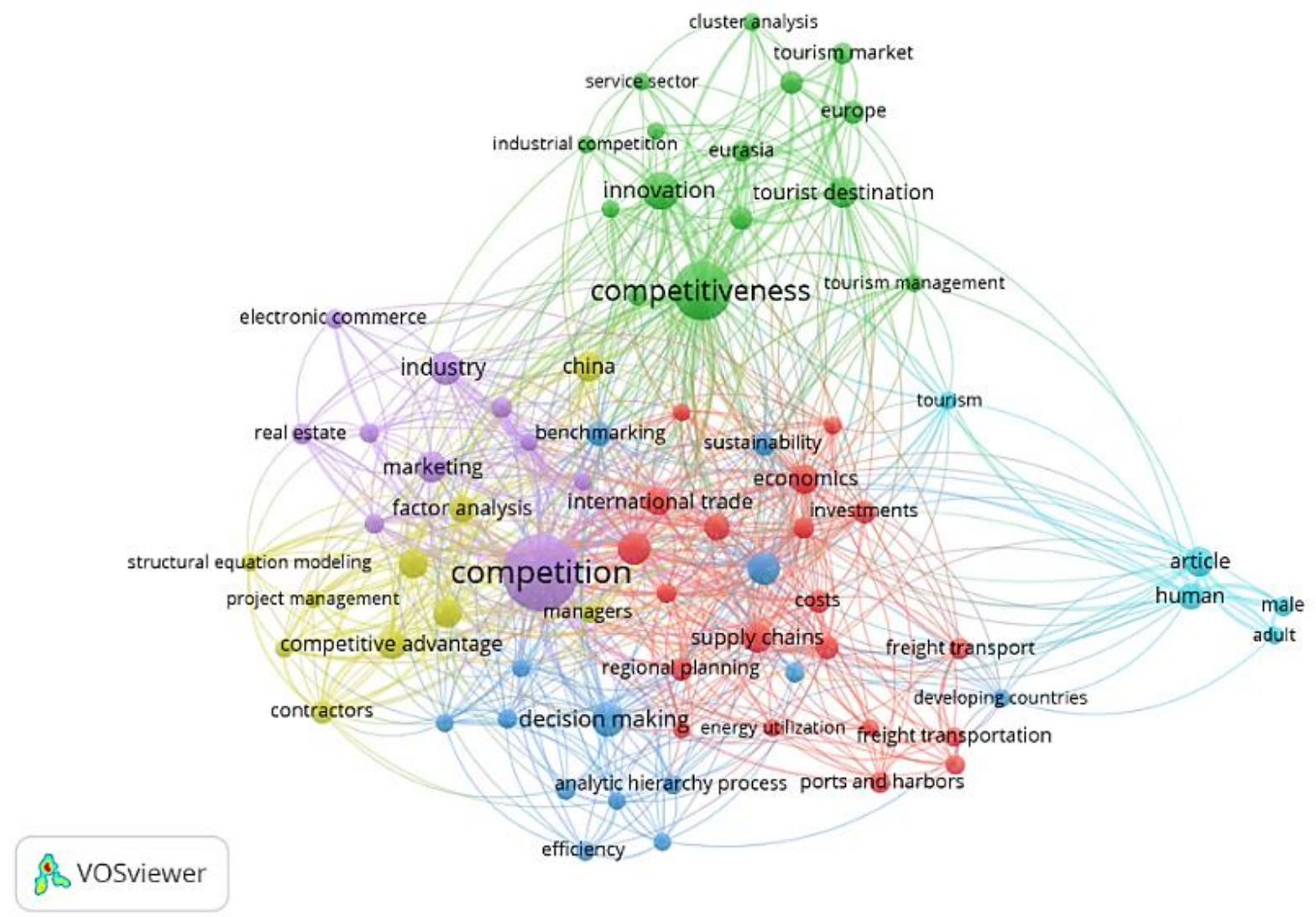

Fig. 3: Nodos de factores de competitividad

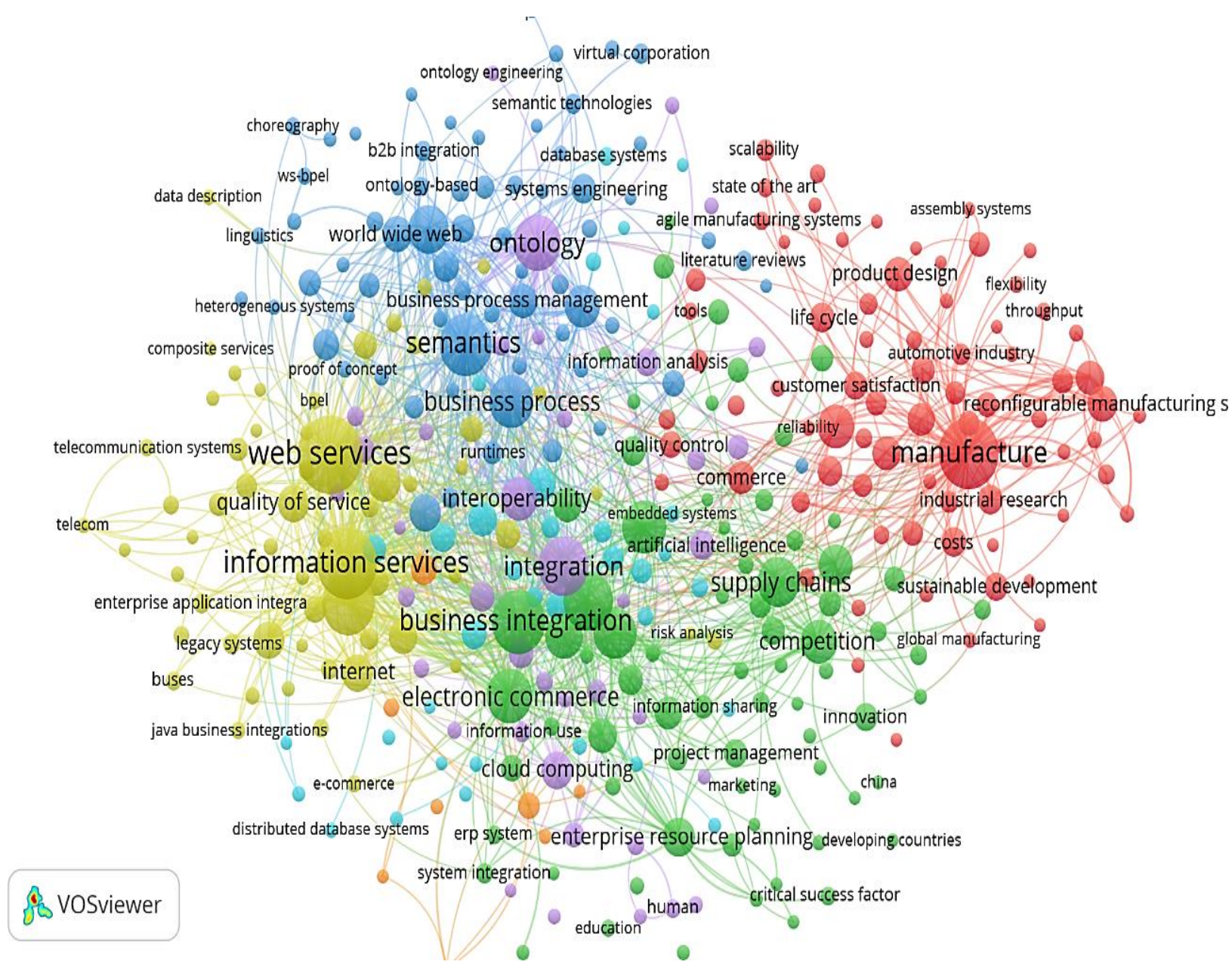

Fig. 4: nodos de factores de integración organizacional 


\section{DISCUSIÓN}

Los resultados de la presente revisión sistemática exploratoria reflejan una tendencia creciente de las investigaciones relacionadas con los factores de competitividad e integración organizacional. Al respecto, Gerasimov et al, (2018) establece que la identificación de los factores de competitividad en las empresas industriales muestra que un direccionamiento prioritario de los procesos, permite el aumento de la eficacia y la calidad. Mientras que Aldana y Bernal, (2018) en la propuesta del modelo de integración organizacional, destaca que toda cadena de abastecimiento debe ser pensada de forma estratégica, integrando los factores de la cultura organización y la gestión del conocimiento.

Adicionalmente, los factores establecidos en la revisión sistemática exploratoria de la competitividad e integración organizacional, se derivan de varios enfoques metodológicos. Específicamente, se presentan factores identificados de estudios a empresas de un sector económico (Manrique y Vargas, 2017), y estudios en los cuales se analizan datos históricos del desempeño de las empresas (Pereira y Crispim, 2015). Adicionalmente, se identifican factores en los diferentes niveles de competitividad propuestos por Labarca (2007): meta, meso, macro y micro. Adicionalmente, en la presente revisión se identifican factores de competitividad desde la perspectiva interna (tecnología de producción innovadora, estrategia de gestión integrada, políticas laborales, capital humano y gestión financiera) y externa de la empresa (cooperación entre empresas, relación con la academia, negociación con proveedores y condiciones de la demanda), de acuerdo con la perspectiva propuesta por Orozco et al. (2014). La revisión destaca la importancia de la gestión del talento humano como un factor relevante para el desempeño óptimo de la empresa, lo cual favorece la innovación en los bienes o servicios que ofertan (Ycaza y Sánchez, 2016). Igualmente, el desarrollo del personal en la organización contribuye a mejorar la eficiencia del negocio integrado (Cherep et al., 2019).

De este modo, los factores identificados corresponden a insumos para el mejoramiento de las capacidades organizacionales, mediante el desarrollo de procesos innovadores que aceleran la capacidad tecnológica, la creación de cadenas de valor productivas y la utilización de provisión de infraestructura de calidad (Pulgarín y Guerrero 2017; Gerasimov et al., 2018). En este contexto, los estudios que se centran en la medición de la competitividad y la integración organizacional en la industria son de gran relevancia debido a que permiten identificar las prioridades que deben ser atendidas por los miembros de la cadena de valor de la empresa (Oaigen et al., 2013). Este premisa tiene especial importancia en las MIPYMES, las cuales deben mejorar continuamente su competitividad para garantizar su sostenibilidad en el mercado (García et al., 2011).

\section{CONCLUSIONES}

La revisión sistemática exploratoria propuesta en el presente artículo permitió explorar la actividad de 23 factores de competitividad y 17 correspondientes a integración organizacional, identificando los países líderes en la producción de la información científica relacionada con las temáticas de estudio. La revisión sistemática exploratoria de la literatura considera artículos publicados en Web of Science, Emerald Insight, Scopus y Ebsco. Los resultados se basan en el análisis a texto completo de 68 artículos originales del periodo comprendido entre el 2013 hasta el mes de mayo del año 2019.

La estructura y cooperación entre empresas, la gestión del marketing, la tecnología de producción innovadora y la estrategia de gestión integrada son los factores más referenciados en materia de competitividad organizacional. Por su parte, la gobernanza de las relaciones estratégicas entre empresas, la coordinación intra-empresarial y la gestión del talento humano corresponden a los factores que favorecen la integración organizacional, de acuerdo con la revisión de la literatura. Estos factores se asocian con los clústeres identificados en cada una de las redes bibliométricas construidas a partir de los registros derivados de la corriente principal de conocimiento en las temáticas de estudio.

Finalmente, este artículo contribuye con la generación de capacidades organizacionales para el desarrollo de la competitividad e integración de procesos; por medio de la identificación de los factores de cada variable estudiada. Por lo tanto, el impacto que genera este tipo de trabajos es la creación y desarrollo de factores y capacidades para el aumento de la competitividad y ejecución de integraciones de manera interna y externa en las empresas. Las limitaciones que se tuvieron para el presente estudio, el tiempo de dedicación para la lectura exhaustiva de cada documento, para el análisis de los diferentes modelos teóricos de las variables estudiadas y de esa manera poder identificar los factores de competitividad e integración organizacional.

\section{REFERENCIAS}

Ahumada, T.E., y Perusquia, J. M. A., Inteligencia de negocios: Estrategia para el desarrollo de competitividad en empresas de base tecnológica. https://doi.org/10.1016/j.cya.2015.09.006, Rev. Contaduría y Administración, 61(1), 127158 (2016). 
Aldana, J. C., y Bernal, C. A., Soft factors in the management of integration supply chains and/or networks: Approximation to a conceptual model, https://doi.org/10.4067/S0718-07642018000200010, Inf. Tecnologica, 29(2), 103-114. (2018).

Becerra, F., Serna, H. M., y Naranjo, C.J., Redes empresariales locales, investigación y desarrollo e innovación en la empresa. Cluster de herramientas de Caldas, Colombia. https://doi.org/10.1016/j.estger.2013.05.013, Estudios Gerenciales, 29(127), 247-257 (2013).

Canales, J. I., y Caldart, A., Encouraging emergence of cross-business strategic initiatives, https://doi.org/10.1016/j.emj.2016.10.003, J. European Management, 35(3), 300-313 (2017).

Cao, Y., y You, J., The contribution of environmental regulation to technological innovation and quality competitiveness An empirical study based on Chinese manufacturing enterprises. Chinese Management Studies, 11(1), 51-71 (2017).

Chandra, C., Kumar, S., y Smirnov, A. V., E-management of scalable supply chains: Conceptual modeling and information technologies framework. https://doi.org/10.1016/s1474-6670(17)38093-x, Hum. Systems Mgmt., 20(2), 83-94 (2001).

Cherep, A., Helman, V., y Lynenko, A., Development of Approaches To Personnel Management Before the Phase of the Merging Process of Enterprises. https://doi.org/10.30525/2256-0742/2019-5-1-233-238, Baltic J. of Economic Studies, 5(1), 233 (2019).

Esser, K., HIllebrand, W., Messner, D., y Meyer-Stamer, J., Competitividad sistémica: Nuevo desafío para las empresas y la política. https://doi.org/10.18356/183846f0-es, Rev. de La CEPAL, 1996(59), 39-52 (1996).

Gerasimov, B. N., Vasyaycheva, V. A., y Gerasimov, K. B., Identification of the factors of competitiveness of industrial company based on the module approach, https://doi.org/10.9770/jesi.2018.6.2(15), Entrep. and Sustainability Issues, 6(2), 677-691 (2018).

Hernández, R., Elementos de Competitividad Sistémica de las PYMES del Istmo Centroamericano. In Naciones Unidas CEPAL, México (2001).

Ibarra, M. A., González, L., y Demuner, M., Competitividad empresarial de las pequeñas y medianas empresas manufactureras de Baja California. https://doi.org/10.21670/ref.2017.35.a06, Est. Fronterizos, 18(35), 107-130. (2017).

Joshi, D., Nepal, B., Singh Rathorey, A.P., y Sharma, D., On supply chain competitiveness of Indian automotive component manufacturing industry. https://doi.org/10.1016/j.ijpe.2012.12.023, Int. J. of Prod. Economics, 143(1), 151-161 (2013).

Khakpour, A., Kazemi, M., Tavakkoli, A., y Fazli, S., Designing a Communicational Model between the Competitiveness Types of Small and Medium Industries in Iran. https://doi.org/10.7232/iems.2018.17.3.550, Ind. Eng. y Mgmt Systems, 17(3), 550-561 (2018).

Labarca, N., Consideraciones teóricas de la competitividad empresarial. Rev.Omnia, 13(2), 158-184 (2007).

Leyva, A. B., Cavazos, J., y Espejel, J. E., Influencia de la planeación estratégica y habilidades gerenciales como factores internos de la competitividad empresarial de las Pymes. https://doi.org/10.22201/fca.24488410e.2018, Cont. y Admon., 63(3), 1-21 (2018).

Mäkinen, H., On the emergence and structure of a new regional network, Rev. Human Systems Mgmt, 20(3), 249-256 (2001).

Manchado, R., Tamames, S., López González, M., Mohedano Macías, L., D’Agostino, M., y Veiga de Cabo., Revisiones sistemáticas exploratorias. Rev. Med. y Seg. Del Trab., 55(216), 12-19 (2009).

Manrique, A., y Vargas, A., Mapa de competitividad en diseño: Validación en empresas del sector lácteo. https://doi.org/http://dx.doi.org/10.18359/rfce.2659 Rev. de La Fac. de Cienc. Econ.: Investigación y Reflexión, XXV(1), 177-202 (2017).

Martínez, M., Construcción de un modelo para facilitar el aprendizaje organizacional. Tesis magister en ingeniería industrial, Universidad de los Andes. Bogotá (2003)

Melgarejo, Z., Vera, M. A., y Mora, E. H., Competitividad de la mipyme y desarrollo regional. Estudio del caso colombiano. Tendencias. Rev. de La Fac. de Cienc. Econ. y Adm., XIV(2), 184-215 (2013).

Messner, D. Latinoamérica hacia la economía mundial: condiciones para el desarrollo de la "competitividad sistémica.", Inst. de Paz y Desarrollo de Duisburg, 1-47 (1996).

Montoya, L. A., Montoya, I. A., y Castellanos, O. F., De la noción de competitividad a las ventajas de la integración empresarial. Rev. Fac. de Cienc. Econ: Inv. y Reflexión, XVI(1), 59-70. (2008).

Nakipova, G. N., Analysis of Competitiveness among Agrarian Companies As a Condition for Stable Development of Kazakhstan. https://doi.org/10.5829/idosi.mejsr.2013, Middle-East J. of Scien. Research, 18(2), 149-153 (2013).

Navarro, O., Ferrer, W., y Burgos, O., La calidad como factor estratégico en el desarrollo competitivo de las Pequeñas y medianas empresas. Univ. y Soc., 10(2), 171-174 (2018).

Oaigen, R. P., Barcellos, J. O. J., y otros 5 autores, Beef cattle production system competitiveness in the South of Brazil. https://doi.org/10.4321/s0004-05922013000200001, Arch. de Zootecnia, 62(238), 161-170. (2013).

Orozco, F., Serpell, A., Molenaar, K., y Forcael E., Modeling Competitiveness Factors and Indexes for Construction Companies: Findings of Chile. https://doi.org/10.1061/(ASCE)CO, J. of Construction Eng. and Mgmt., 140(4), 556-563. (2014). 
Pereira, I. L., y Crispim, S. F., Fatores explicativos da alta competitividade no longo prazo: presença dos fatores identificados nas empresas norte-americanas em empresas da BM \& FBOVESPA. https://doi.org/10.1590/0104530X1405-14 Gestão \& Produção, 22(1), 82-95 (2015).

Porter, M. E., Estrategia competitiva, $1^{\text {ra }}$ Ed., 1-550, CECSA. Tlalpan, Mexico (1987).

Pulgarín, S. A., y Guerrero, N. A., Innovation and competitiveness: organizational culture and business model. https://doi.org/10.15665/rde.v15i2.1023, Dimensión Empresarial, 15(2), 25, (2017).

Ramírez, J. V., Factores éticos que influyen en la competitividad de las empresas en el departamento del Quindío, Colombia. Rev. de Admon. y Neg., 32(55), 59-71 (2016).

Rojas, P., ¿Que es la competitividad?, Rev. Com. En La Agricultura, 9, 4-24 (1999).

Ryzhkova, E., y Prosvirkin, N., Cluster initiatives as a competitiveness factor of modern enterprises, European Research Studies Journal, 18(3), 21-30 (2015).

Saavedra, L. M., y Tapia, B., Mejores prácticas y factores de competitividad en las micro, pequeñas y medianas empresas mexicanas., Rev. Economía, 0(32), 11-36 (2011).

Song, G., Song, S., y Sun, L., Supply chain integration in omni-channel retailing: a logistics perspective. https://doi.org/10.1108/IJLM-12-2017-0349, Int. J. of Log. Mgmt, 30(2), 527-548 (2019).

Tambade, H., Singh, R. K., y Modgil, S., Identification and evaluation of determinants of competitiveness in the Indian autocomponent industry. https://doi.org/10.1108/BIJ-09-2017-0260, Benchmarking, 26(3), $922-950$ (2019).

Todeva, E. y Knoke, D, Strategic alliances and models of collaboration. https://doi.org/10.1108/00251740510572533, Management decision. 43(1), 123- 148 (2005).

Vargas, A. F., Rech, I., y Santos, S. A., Fatores de competitividade empresarial em empresas instaladas em um parque tecnológico brasileiro. https://doi.org/10.20397/g\&amp;t.v16i2.869, Rev. Gest. y Tecn., 16(2), 100-126 (2016).

Verner, T. National competitiveness and expenditure on education, research and development. Journal of Competitiveness, 3(2) (2011).

Ycaza, D. E., y Sánchez, A. M., Factores de competitividad orientados a la pequeña y mediana empresa (PYME) en Latinoamérica: revisión de la literatura. Rev. San Gregorio, (15), 104-111 (2016). 
